\title{
The Status of the DELPHI Very Forward Ministrip Detector
}

\author{
April 30,1996
}

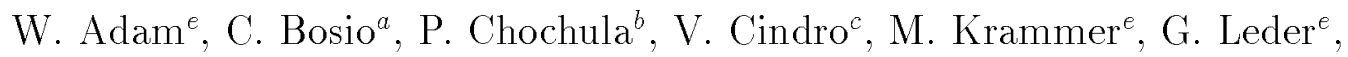
C. Mariotti ${ }^{a}$, J. Masik ${ }^{d}$, E. Margan ${ }^{c}$, N. Neufeld ${ }^{e}$, H. Pernegger ${ }^{e}$, M.

Pernicka $^{e}$, D. Rakoczy ${ }^{e}$, V. Rykalin ${ }^{a}$, D. Žontar ${ }^{c}$

${ }^{a}$ INFN sezione Sanita, Roma

${ }^{b}$ Comenius University of Bratislava,SLQ-84215 Bratislava

${ }^{c}$ Institut Jožef Stefan, Ljubljana, Slovenija

${ }^{d}$ Nuclear Centre of MFF, Charles University,Praha,Czech Republic

${ }^{e}$ Institut für Hochenergiephysik d. Österr. Akademie d. Wissenschaften, Wien, Austria

Contribution to the $6^{\text {th }}$ International Conference for Experiments at $e^{+} e^{-}$-Colliders, Novosibirsk, Russia, Feb. 1996

\begin{abstract}
We present the results of final acceptance tests of the DELPHI VFT ministrip detector before and during detector assembly. Dedicated tests were carried out to detect substandard channels, measure the $\mathrm{S} / \mathrm{N}$ performance and test the assembled detector for electronic failures.
\end{abstract}




\section{Introduction}

The DELPHI detector is being upgraded by a new silicon tracker in the very forward region to fulfill the physics demands of the LEP200 program [1]. The VFT detector enhances the tracking capabilities of the DELPHI detector in the forward region by two end-cap-like segments. It covers the angular region between $10^{\circ}$ and $25^{\circ}$ with respect to the beam axis and features a two dimensional readout. The severe space restrictions in this region lead us to develop a new arrangement of detectors and electronic hybrids. The layout of the detectors was optimized with prototypes. Final acceptance tests on the VFT ministrip modules have been carried out before the detector installation in May 1996.

\section{The layout of the VFT detectors}

The VFT was designed to provide stand alone recognition of track segments in front of the DELPHI forward tracking system. The layout had to take into account the severe limitations in space available for the active detector material as well as for the electronics and the support system [2-4]. Each side consists of two planes of "macro" pixel detectors followed by two planes of ministrip detectors with $200 \mu \mathrm{m}$ read out pitch. In the following we will describe only the ministrip detector. Each ministrip plane consists of two half rings with 6 detector modules each surrounding the beam pipe. Figure 1 shows the arrangement of the detector modules. Each VFT detector module is formed by two single-sided strip detectors, called TOP and BOTTOM, which are glued together back to back with perpendicular strip orientation. The square detector shape allows us to use one single layout for the two module sides. A four degree stereo angle between the corresponding strips of two planes suppresses ambiguities. Due to the limitation in space, the electronic hybrid is glued directly onto the active detector area as shown in figure 1. Each silicon detector has $200 \mu \mathrm{m}$ read out pitch with one intermediate strip, which is not directly connected to an amplifier channel. The use of intermediate strips, which are read out via their inherent interstrip capacitance to read out strips, allows to keep the total number of electronic channels low, fulfilling the requirements of spatial resolution, cost and power consumption. Testbeam measurements indicate an intrinsic spatial resolu- 
tion in the detector plane of 10 to $30 \mu \mathrm{m}$ depending on the track inclination $[5,6]$

\section{Acceptance tests of the VFT ministrip mod- ules}

The aim of the tests is to detect any electronics problem on the detector, calibrate the signal gain for each channel, record the number of dead, noisy and saturated channels and verify the $\mathrm{S} / \mathrm{N}$ performance and the time stability of detector parameters, like the average r.m.s. noise.

To test these parameters we constructed a test setup which allowed to test both individual modules and fully assembled planes with a calibration signal and a Sr-90 source. In the backplane calibration setup, shown in figure 2, a voltage step $V_{0}$ is generated externally and coupled to the backplane voltage supply line of the module. The detector is fully depleted, therefore the backplane capacitance of each strip acts as a calibration capacitor on the amplifier input. The input charge equals to $C$ (backplane $) \times V_{0}$ and corresponds approximately to the charge deposited by a minimum ionizing particle. In the test setup only genuine components of the VFT detector system are used in order to obtain test results which are comparable to final operating conditions. Furthermore the use of final electronics, cables and data aquisition allows the optimisation of system parameters.

\section{Measurement results}

During the measurements three types of channel defects were observed: Strips are regarded as "dead" if their response is less than $50 \%$ of the average signal of all channels of the chip. Channels with a r.m.s. noise higher than 5 times the average r.m.s. noise of all channels are regarded as "noisy", channels with a pedestal voltage saturating the SIROCCO ADC are called "saturated". A VFT ministrip module is accepted if the total number of defect channels does not exceed 5 channels on each module side, i.e. $2 \%$. The number of defect channels per TOP and BOTTOM detector of 31 modules is plotted in figure 3. On average each module side has two bad channels. The number of dead and noisy channels is approximately the same, while only very few 
channels were saturated. The total number of defect channels is 195 out of 25376 channels, i.e. $0.8 \%$. The dead and noisy channels measured on the assembled ministrip planes correspond exactly to the defect channels detected in the single module test. No further defect channels appeared during the detector assembly. Figure 4 shows the result of the Sr-90 test on a single VFT ministrip module. The two plots on the left show the $\mathrm{S} / \mathrm{N}$ distribution for the TOP and BOTTOM detector. The most probable $\mathrm{S} / \mathrm{N}$ measured is 32 on both detectors. The central plots give the r.m.s. noise versus the channel number. The plot on the right shows the hit position on the TOP detector versus the hit position on the BOTTOM detector. Only a part of the detector is irradiated by the collimated source. The presence of the electronic hybrid and other components on the detector screen the low energy electrons of the source, which results in a non-uniform hit distribution in the source test. The most probable signal-to-noise ratio measured on all modules is plotted in figure 5. During the 21 hours stability test, the noise level was monitored and found to be stable in time. No additional noise due to the long signal transfer lines has been observed during the tests. Furthermore we did not observe crosstalk between the two sides of the module. Crosstalk between the control signal lines on the hybrid and the read out lines of detector is avoided by a careful routing of the relevant control lines on the hybrid.

\section{Conclusions}

A new silicon tracking detector for the very forward region of the DELPHI experiment was tested before the final installation. On average each detector has two defect channels which is equivalent an overall efficiency of $99.2 \%$. The number of dead and noisy channels did not show a time dependence during the stability tests. The signal-to-noise ratio as measured on all detectors is sufficiently high to allow safe and reliable operation.

\section{References}

[1] Proposal for the DELPHI Very Forward Tracker,DELPHI 93-52

[2] C.Bosio et al., Nucl.Instr. and Meths. A 360(1995)71-74 
[3] V.Cindro et al., Nucl.Phys. B (Proc. Suppl.)44 (1995)292-295

[4] A.Andreazza et al., Nucl.Instr. and Meths. A 367(1995)198-201

[5] P. Chochula et al., DELPHI internal note DELPHI 94-44 Track 78

[6] H. Pernegger, PhD thesis, Technical University Vienna, 1996

[7] J.C. Stanton, IEEE Trans. Nucl. Sci. Vol. 36, No. 1 


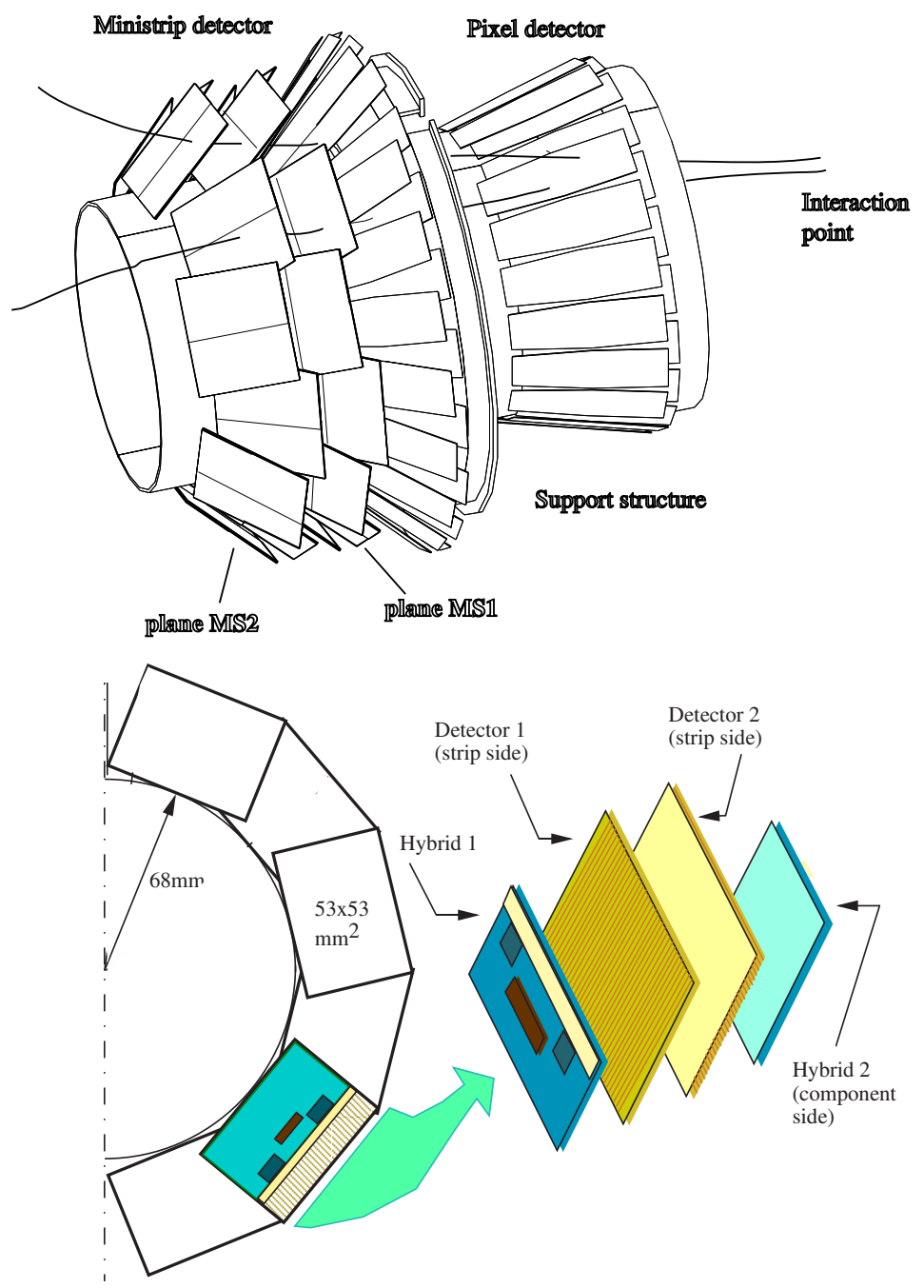

Figure 1: Planes of the DELPHI Very Forward Tracker Ministrip detector 

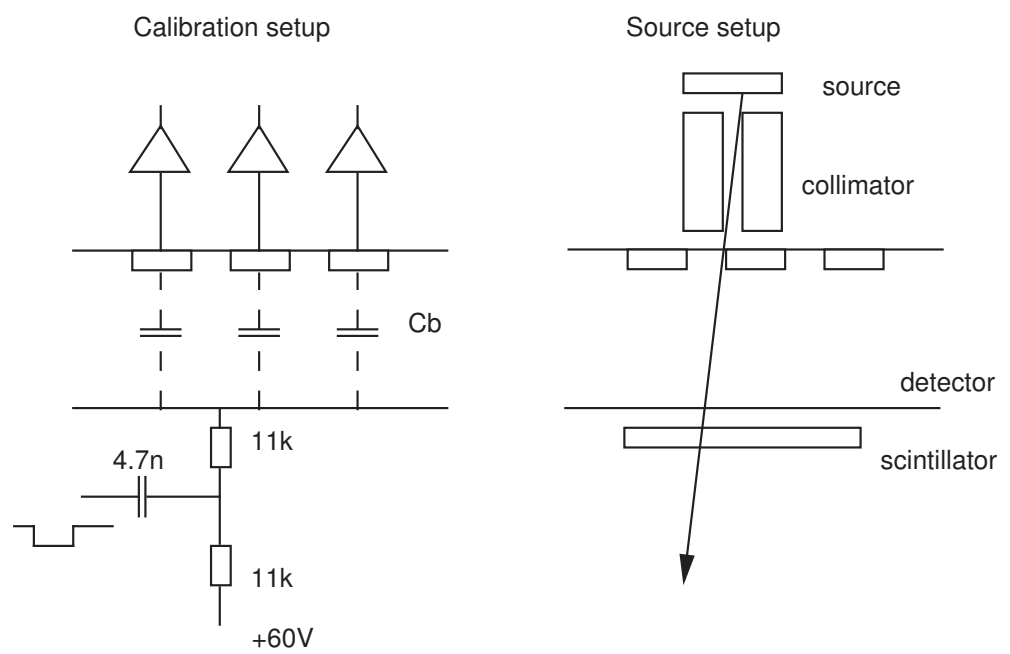

Figure 2: Schematic view of the test setup
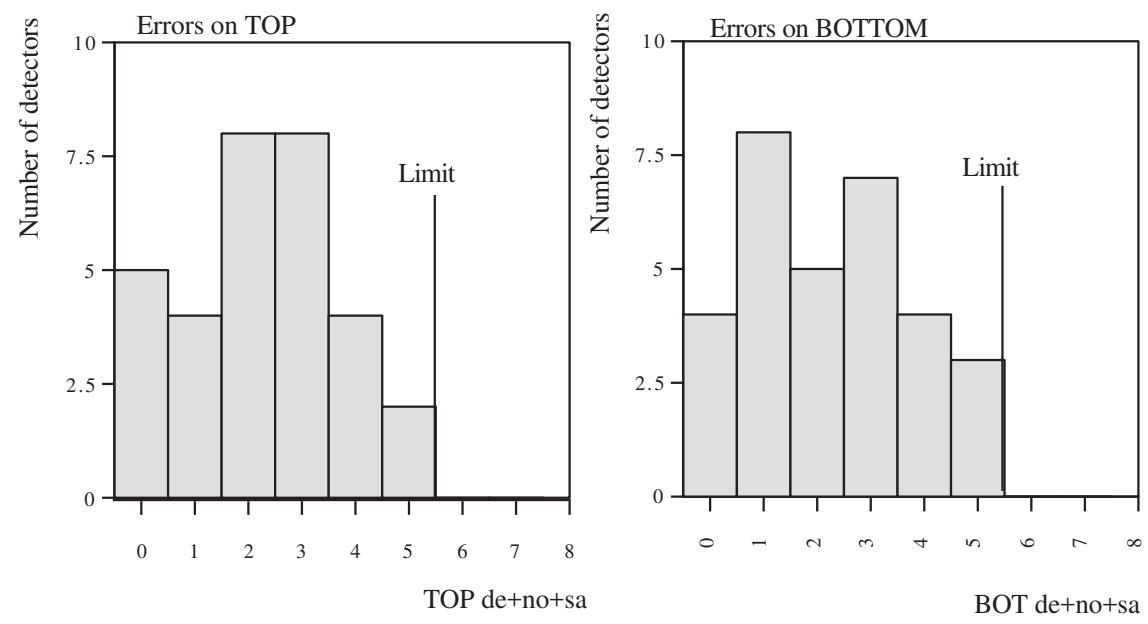

Figure 3: Number of defect channels on TOP and BOTTOM detector 


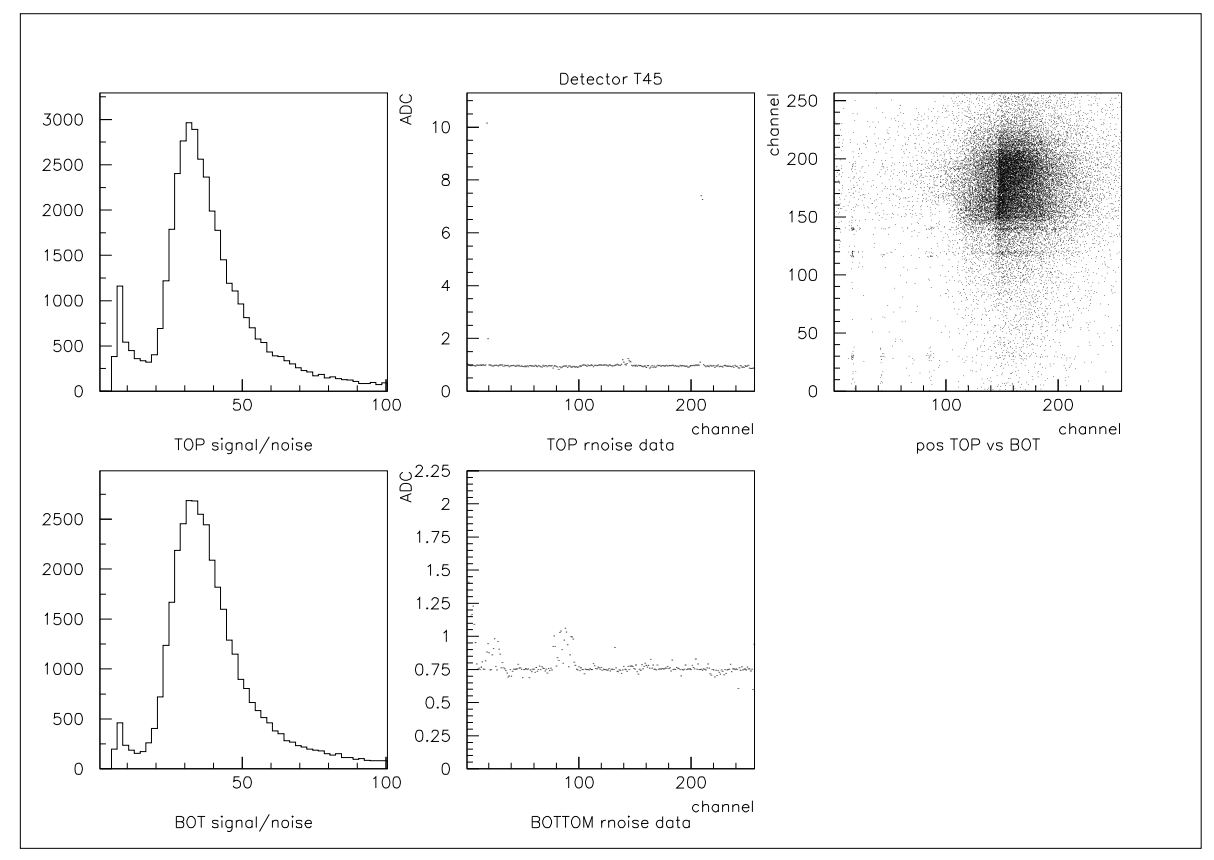

Figure 4: Source test results on one VFT ministrip module 
detector statistics

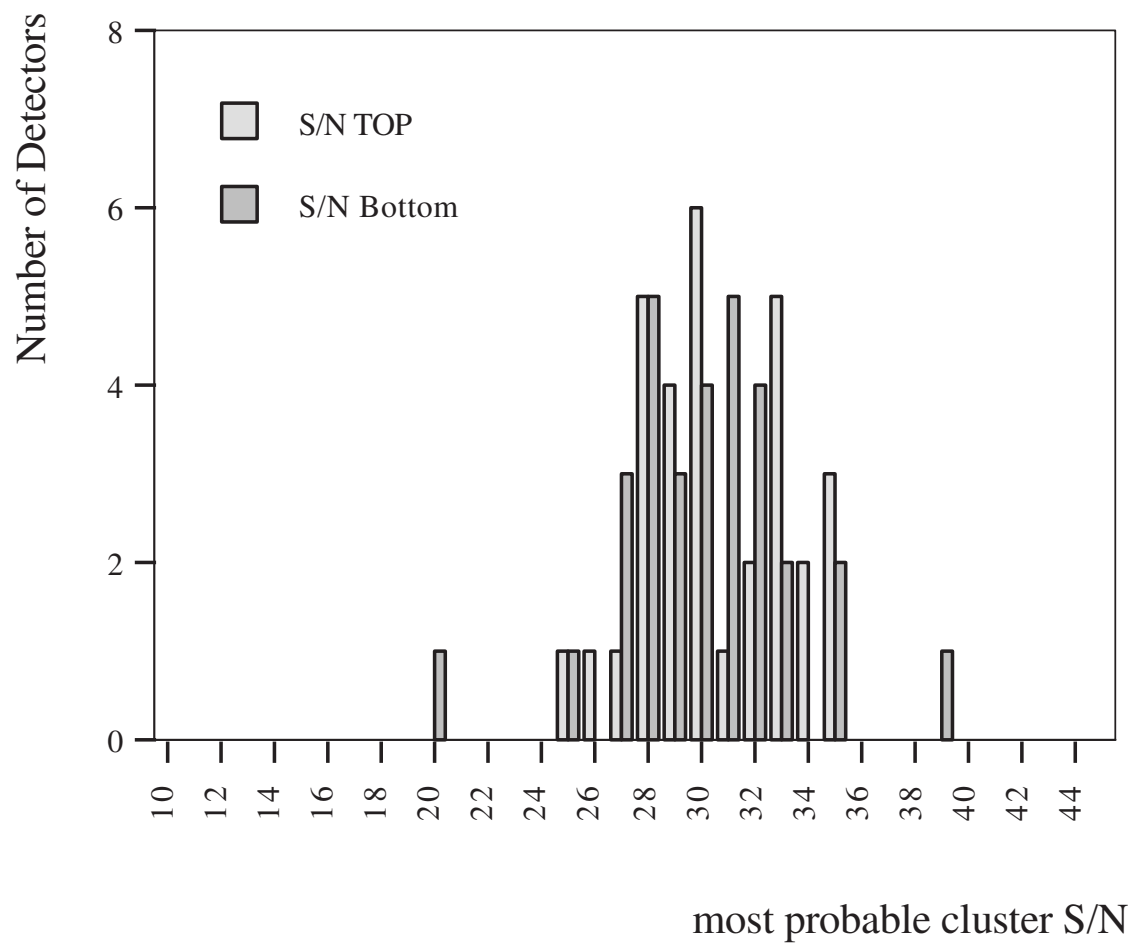

Figure 5: Distribution of most probable $\mathrm{S} / \mathrm{N}$ for TOP and BOTTOM detector 